Journal of Patient-Centered

\title{
Multiracial Patient Experiences With Racial Microaggressions in Health Care Settings
}

Cyndy R. Snyder

Prince Z. Wang

Anjali R. Truitt

Follow this and additional works at: https://aah.org/jpcrr

Part of the Bioethics and Medical Ethics Commons, Health and Medical Administration Commons, Health Services Research Commons, Medical Education Commons, and the Medical Humanities

Commons

\section{Recommended Citation}

Snyder CR, Wang PZ, Truitt AR. Multiracial patient experiences with racial microaggressions in health care settings. J Patient Cent Res Rev. 2018;5:229-38. doi: 10.17294/2330-0698.1626

Published quarterly by Midwest-based health system Advocate Aurora Health and indexed in PubMed Central, the Journal of Patient-Centered Research and Reviews (JPCRR) is an open access, peer-reviewed medical journal focused on disseminating scholarly works devoted to improving patient-centered care practices, health outcomes, and the patient experience. 


\title{
Multiracial Patient Experiences With Racial Microaggressions in Health Care Settings
}

\author{
Cyndy R. Snyder, PhD, ${ }^{1}$ Prince Z. Wang, ${ }^{2}$ Anjali R. Truitt, PhD, MPH ${ }^{2}$ \\ ${ }^{1}$ Department of Family Medicine, University of Washington, Seattle, WA; ${ }^{2}$ Surgical Outcomes Research Center, \\ Department of Surgery, University of Washington, Seattle, WA
}
Purpose Illuminating patients' experiences of microaggressions in health care settings can help practitioners develop care that is more culturally responsive. While much of the literature on health care disparities focuses on minority groups generally, we sought to identify and to describe the ways in which racial microaggressions manifest for multiracial individuals and families specifically.

Methods Using a combination of interviews and focus groups, we conducted 15 interviews and 3 focus groups. Eligible participants self-identified as more than one race and/or they self-identified as part of an interracial family, and they and/or someone they considered to be part of their family received health care in the past 12 months. We performed a content analysis to describe the dominant ways racial microaggressions presented in health care interactions.

Results A total of 31 participants shared their experiences in health care settings, including their experiences with racism, racial bias, and microaggressions. Based on their experiences, we describe 6 prevalent microaggressions: mistaken identity, mistaken relationships, fixed forms, entitled examiner, pervasive stereotypes, and intersectionality. Many acknowledged their experiences with racial microaggressions in health care were similar to those they experienced in everyday settings; however, the power dynamics differed in health care.

Conclusions Understanding patient perspectives about racial microaggressions suggests opportunities to improve patient-provider communication. For multiracial individuals and families, racial microaggressions may have implications for patient engagement in health care. Findings of this study highlight implications for theory and research as well as opportunities to facilitate systematic improvements in the provision of culturally responsive health care services. (J Patient Cent Res Rev. 2018;5:229-238.)

Keywords racism; minority health; family; health care disparities; culturally responsive care

I n 2002, the Institute of Medicine's landmark report, Unequal Treatment: Confronting Racial and Ethnic Disparities in Healthcare, ${ }^{1}$ concluded that health care provider bias, prejudice, and stereotyping in part contributes to racial and ethnic health care disparities. The work highlights how health care providers can express negative racial attitudes and stereotypes, which can manifest in prejudicial behaviors and treatment.

Correspondence: Cyndy R. Snyder, PhD,

University of Washington, 4311 11th Ave NE, Suite 210, Seattle, WA 98105 (snyderc@uw.edu)
Since this seminal report, health disparities research continues to show that health care providers exhibit racial bias and that patients of color experience various forms of racism in health care settings. ${ }^{2}$

Such biases include racial microaggressions, a more subtle and cumulative act of everyday racism. Psychiatrist Chester Peirce initially coined the concept of racial microaggressions in the 1970s, ${ }^{3,4}$ and since then, scholars have expanded the concept. Pérez Huber and Solórzano provided a comprehensive definition of racial microaggressions, ${ }^{5}$ one that incorporates individual-level racism as well as the larger systems of 
racism that serve to marginalize and denigrate people of color: "A form of systemic, everyday racism used to keep those at the racial margins in their place. [Racial microaggressions] are: (1) verbal and non-verbal assaults directed toward People of Color, often carried out in subtle, automatic or unconscious forms; (2) layered assaults, based on race and its intersections with gender, class, sexuality, language, immigration status, phenotype, accent, or surname; and (3) cumulative assaults that take a psychological, physiological, and academic toll on People of Color." 5

Scholars have begun to explore the nature of racial microaggressions in the health care context, noting that such experiences have the potential to poorly impact health outcomes. ${ }^{2,6}$ For example, Walls and colleagues focused on racial microaggressions among Native Americans living with a chronic disease and found that experiences with microaggressions in health care settings contributed to worse mental and physical health reports. ${ }^{2}$

Yet, much of the literature on racism and racial microaggressions in health care focuses on people of color generally assuming a monoracial perspective and categorization, overlooking the experience of multiracial people and families. Multiracial individuals - those who identify with more than one racial or ethnic group - are one of the fastest growing U.S. subpopulations, growing three times faster than the general population. ${ }^{7}$ In the 2010 Census, roughly 7.5 million Americans selected more than one racial category, and this is expected to more than triple by $2060 .^{8}$

While many of the racialized experiences of multiracial people are similar to those experienced by people of color more broadly, research outside the health care setting suggests that multiracial people and families have unique racialized experiences. ${ }^{9}$ Pew Research Center found that more than half of multiracial people $(55 \%)$ report that they experience racial slurs or jokes and nearly one-quarter (24\%) express annoyance about assumptions regarding their racial background. ${ }^{7}$ Although these experiences often reflect racism experienced by people of color in general, for multiracial people and families, racialized interactions may be complicated due to questions about their racial identity, background, and family position. Health care settings may be particularly charged if health care professionals consciously or unconsciously communicate racial biases to racially mixed families. ${ }^{10}$

Because of their cross-racial dynamics, multiracial individuals and families offer a unique lens to examine the impact of racial bias on health care disparities. Using a combination of interviews and focus groups, we identify and describe the ways in which racial microaggressions manifest in health care settings for multiracial individuals. In an effort to better center patient experiences, this work focuses specifically on identifying racial microaggressions and racism from the patient perspective. Our goal was to identify aspects of racism and racial microaggressions in health care settings and offer a more complex intersectional view of race that might not be fully understood in light of previous research, theory, and methods rooted in monoracial assumptions about race and family.

\section{METHODS \\ Participants}

We recruited participants using a combination of purposive and snowball sampling. ${ }^{11}$ We identified potential participants passively by circulating information about the research study at national conferences, on multiracial organization websites and listservs, through social media, and through word of mouth. Interested participants completed an electronic screening survey. Participants were eligible if 1) they self-identified as more than one race and/or selfidentified as part of an interracial family relationship, and 2) they or someone they considered to be part of their family had received health care in the past 12 months. We contacted eligible participants and offered them the opportunity to join 1) an in-person interview, 2) a video conferencing or telephone interview, or 3) a web-based focus group. Recruitment continued until thematic saturation was reached. This study received an exempt determination from the University of Washington (Seattle, WA) institutional review board.

A total of 66 eligible participants indicated interest in either the interviews or focus groups. Of these, 40 consented (response rate: 61\%) and 31 participated (participation rate: $78 \%$ ). Participants were split approximately evenly between 3 focus groups (comprising a total of 16 participants) and 15 interviews. Interviews were conducted in-person at a location the 
participant preferred or by using an institutionally approved web-conferencing program. This allowed participants to select a venue that best fit their needs.

\section{Data Collection}

From February 2017 to June 2017, we collected information from participants using a combination of interviews and focus groups. We chose to conduct both focus groups and interviews to allow participants to select the venue that was most comfortable and convenient for them, recognizing that some individuals may be more comfortable sharing their stories in a group format than others. For consistency across data collection, we used a guide to direct the conversations. The guide supplied general topics and questions intended to elicit each participant's experience of racism, racial bias, and microaggressions in the health care setting. Participants were allowed to discuss all relevant or formative experiences, although we grounded the conversations in health care experiences occurring within the past 12 months. Each interview or focus group began in the same way by asking participants to share their experiences using health care services. From there, participants were asked more detailed questions about the role of race in their health care interactions, their health-seeking preferences, and how they felt their position as a multiracial person or as part of a multiracial family shaped their experiences. Participants also were asked questions about how they dealt with negative interactions and what suggestions they had for addressing racial bias in health care settings. The guide was designed to elicit both direct and indirect, or vicarious, experiences of racism. We chose this approach because scholars have suggested that vicarious racism - experiences that one observes or hears about but does not personally experience themselves, such as racism experienced by a friend, colleague, or relative - has the potential to cause stress similar to that induced by direct experiences. ${ }^{12}$

For interviews, a trained interviewer (A.R.T. or C.R.S.) conducted the semi-structured interview. We audiorecorded and transcribed the interviews, then reviewed the transcripts for accuracy and context. For focus groups, a trained moderator (C.R.S.) posed questions using an online forum-style platform designed specifically for gaining feedback from groups. Participants responded in writing to the questions and were encouraged to respond to other participants' comments. Although focus group participants were encouraged to reflect and comment on each other's responses, most did not, and the resulting responses were more similar to individual interviews than interactive focus groups. We formatted responses by group and by individual to evaluate within- and acrossgroup similarities and differences.

\section{Data Analysis}

We analyzed data using an inductive grounded theory approach, identifying themes that emerged from the data. ${ }^{13,14}$ To ensure the research was conducted and analyzed in a rigorous way, analysis followed an interpretive, multiphased approach. ${ }^{15}$ All participant responses were reviewed, and an initial coding schema based on response frequency and patterns across responses was developed. We reviewed the focus group and the interview data separately, applying a withincase and across-case approach. ${ }^{16}$ The content across data collection strategies was similar, despite focus group participants' ability to read and respond to other group members' responses. Unlike traditional focus groups, in which the group dynamic is inseparable from the conversation, participants in the web-based focus group answered the questions independently and researchers exported each individual's responses. Therefore, we opted to analyze the individual focus group responses and the interview transcripts using the same process. Through an iterative, reflexive process, the research team added, refined, and defined codes. We employed a content analysis ${ }^{17,18}$ to identify instances when participants described racism, racial bias, and microaggressions.

We imported de-identified participant responses into Dedoose (SocioCultural Research Consultants, LLC, Manhattan Beach, CA), a computer assistive qualitative analysis software. ${ }^{19}$ Two researchers (A.R.T. and P.Z.W.) applied these codes to each participant response independently. We compared our coding application, identifying similarities and differences, and communicated regularly about the codes and coding applications. Discrepancies were reconciled through consensus. To enhance validity, we consulted with our Stakeholder and Patient Advisory Group (consisting of patients, clinicians, and scholars) about emerging themes. 


\section{RESULTS \\ Participant Characteristics}

The majority of participants identified with more than one racial category (94\%). As Table 1 shows, participants also were mostly female $(81 \%)$ and had postsecondary education (75\%).

Participants described that the racial microaggressions they faced in health care were similar to those they experienced every day in other settings. We did not specifically ask participants to indicate the race of the provider in the interaction because, often, they discussed multiple interactions with various providers. However, in sharing their stories, the majority of participants referenced experiences with white providers and described the difficulty in locating providers from racially diverse backgrounds. As Table 2 highlights, 6 prevalent microaggressions were identified: mistaken identity, mistaken relationships, fixed forms, entitled interrogator, pervasive stereotypes, and intersectionality.

\section{Mistaken Identity}

Many of the participants expressed instances in which health care providers assumed they were one race or they were documented as such in medical records, despite self-identifying as more than one race. A multiracial participant who identified as mixed race, Asian and white, said, "Recently in the online patient portal I noticed that my race was listed as white. I was so mad! I emailed them saying I can only assume that they assigned me white based on my appearance."

Another participant articulated that the mistaken identity made her question whether the medical results were her own:

Table 1. Participant Demographics

\begin{tabular}{|c|c|c|c|}
\hline Characteristic & $\begin{array}{c}\text { Interview } \\
(n=15)\end{array}$ & $\begin{array}{c}\text { Focus group } \\
(n=16)\end{array}$ & $\begin{array}{c}\text { Total } \\
(n=31)\end{array}$ \\
\hline Age, mean in years & 29 & 33 & 31 \\
\hline Those reporting more than 1 racial category & $13(87 \%)$ & $16(100 \%)$ & $29(94 \%)$ \\
\hline \multicolumn{4}{|l|}{ Gender } \\
\hline Female & $10(67 \%)$ & $15(94 \%)$ & $25(81 \%)$ \\
\hline Male & $3(20 \%)$ & $0(0 \%)$ & $3(10 \%)$ \\
\hline Gender queer/nonbinary & $2(13 \%)$ & $1(6 \%)$ & $3(10 \%)$ \\
\hline \multicolumn{4}{|l|}{ Education } \\
\hline Some college, associate's degree, or post-HS certificate & $4(27 \%)$ & $4(25 \%)$ & $8(26 \%)$ \\
\hline Four-year college graduate & $5(33 \%)$ & $2(13 \%)$ & $7(23 \%)$ \\
\hline Professional or graduate degree & $6(40 \%)$ & $10(63 \%)$ & $16(52 \%)$ \\
\hline \multicolumn{4}{|l|}{ Health care seeking (in the past 12 months) } \\
\hline Those who sought care for themselves & $14(93 \%)$ & $16(100 \%)$ & $30(97 \%)$ \\
\hline Primary care & $10(67 \%)$ & $14(88 \%)$ & $24(77 \%)$ \\
\hline Emergency/urgent care & $4(27 \%)$ & $6(38 \%)$ & $10(32 \%)$ \\
\hline Specialty care & $8(53 \%)$ & $7(44 \%)$ & $15(48 \%)$ \\
\hline Those who accompanied an immediate family member & $7(47 \%)$ & $8(50 \%)$ & $15(48 \%)$ \\
\hline Primary care & $6(40 \%)$ & $6(38 \%)$ & $12(39 \%)$ \\
\hline Emergency/urgent care & $6(40 \%)$ & $1(6 \%)$ & $7(23 \%)$ \\
\hline Specialty care & $3(20 \%)$ & $1(6 \%)$ & $4(13 \%)$ \\
\hline
\end{tabular}

HS, high school. 
Table 2. Multiracial Microaggressions in Health Care

\begin{tabular}{|c|c|}
\hline Theme & Description \\
\hline Mistaken identity & - When an assumption is made about the patient's race \\
\hline Mistaken relationships & -When an assumption is made about family makeup \\
\hline Fixed forms & - When forms only allow for selection of one racial category or "Other" \\
\hline Entitled examiner & $\begin{array}{l}\text { - When providers ask probing questions outside what feels necessary for medical care related to } \\
\text { race or racial stereotypes } \\
\text { - When providers ignore race and culture, or their potential impact on health is not communicated }\end{array}$ \\
\hline Pervasive stereotypes & $\begin{array}{l}\text { - When providers hold low expectations about an individual seemingly due to their racial } \\
\text { background or make assumptions about educational attainment, insurance, or finances }\end{array}$ \\
\hline Intersectionality & $\begin{array}{l}\text { - When participants identified microaggressions or feelings of awkward encounters in health } \\
\text { care that they could not attribute to just race }\end{array}$ \\
\hline
\end{tabular}

"This relates to an experience I had approximately 10 years ago where I went in for an HIV AIDS test. I returned for my results (as is required) and was sitting with the provider who reported my results (negative) and pointed to the form where the results were listed. I noticed on the form that I was identified as a 'white female.' I was perplexed and assumed that the results that were reported on the form were not meant for me as I did not identify myself as white. I did not say anything to the counselor and left the meeting assuming she gave me the wrong results and I might really be HIV-positive."

These quotes highlight how assumptions about one's race, or mistakes in the medical records, can have broader impact for individual patients.

\section{Mistaken Relationships}

Many participants mentioned times when they presented to a clinic or hospital with family members, yet staff assumed they were not related. For example, one participant recalled their experience when accompanied by their parent:

"I remember the discomfort that my dad, when he would take me into the doctor when I was younger, that they'd [clinic staff] be like, 'Oh, is this your new adopted child? What's your relationship to this child?' And, it's such an awkward moment for him and myself."

This participant expressed feeling uncomfortable and awkward because of questions from staff.
More broadly, the experience reflects an underlying assumption that family members should look alike and be of the same race, and when they do not, then adoption is seemingly the only plausible explanation. Another participant described the impact of such assumptions on their care:

"I feel like the fact that my family members aren't seen as being family and being truly related to me takes away from my ability to see them as a support system, because instead I'm seeing them as a choice I have to defend. Yes, this is my mom, I did bring her because she's related to me and I love her, and I need her to help me."

\section{Fixed Forms}

Many participants reported difficulty self-identifying with more than one race category on medical forms. As one participant mentioned, "Although many providers have updated their [intake forms], some still don't provide an option to check multiracial/mixed-race." Most stated that they would prefer to select each of the racial categories with which they identify, but the option to select multiracial is a better alternative than having to select only one race category. Several participants noted the importance of this not only for their own health care management but also for research, as medical records are increasingly used for this purpose.

\section{Entitled Examiner}

Participants described how the relationship between patient and provider differs from other relationships. 
While patients and providers both ask informationseeking questions, the social dynamic in clinic settings is such that providers may ask questions about race and ethnicity, gender and sexuality, and health behaviors that in other social contexts may be considered inappropriate. Providers' questions may be wellintentioned and even necessary to guide treatment, and participants learned to expect such questions as part of receiving health care.

One participant in an interracial marriage with young multiracial children described his experiences accompanying his family to medical appointments: "There's always a question as to 'Are you the father type of thing, or are you the husband?' And I don't know what that means? You know, I'm assuming they're just asking to find out who I am. I would be lying if in the back of my mind I didn't wonder what they if there's anything else to it." This sentiment parallels other everyday racial microaggressions reported by multiracial individuals and families. . $^{90,21}$

Providers by and large controlled the conversation, even around race and culture. Participants mentioned being asked invasive questions that felt related to provider biases. Several participants mentioned being asked about blood quantum. For example, one participant stated, "I also tend to get the question of 'how much' native I am, but I'm never asked how much of anything else I am." Participants reported having difficulty distinguishing questions rooted in provider curiosity from those of medical need and did not feel empowered to not respond. While some of the questions related to health behaviors, like substance use, gambling behaviors, and reproductive decisionmaking, participants described feeling that they were asked specific questions because of their racial background and/or provider's racial stereotypes.

Most interviewees identified race and culture as important to their lives and suggested that these identities impacted their health and health behaviors in different ways. Several participants expressed disappointment or surprise that primary care providers did not engage them in conversations about their race and culture. As one participant noted, culture is infrequently engaged with in health care:
"I just feel like it [culture] is such an important part to my experience and to my kids' experience of how they experience the world; but, it's never brought up in our medical conversations. And, so, it does just feel like it's an ignored part, and like we are sort of just all supposed to be the same, without this acknowledgment that there are differences."

Yet, few participants referenced providers engaging them in an open-ended conversation about their culture and its impact on their health. Additionally, some participants noted that race and culture may not be relevant to all medical encounters, particularly emergency or urgent care.

\section{Pervasive Stereotypes}

Participants expressed feeling that providers made assumptions about their education, insurance, or financial resources due to racial bias or stereotypes in the health care setting. They described that this resulted in a shift in how the provider or staff communicated with them. As one participant described, patient-provider communication changes with provider awareness about higher educational attainment:

"I think I've had numerous times, and I don't know if this is race or age or what, but numerous times people are all of a sudden, 'Oh, you have a Ph.D.?' and then, all of a sudden, they feel like they can talk to me in a completely different way about what's going on with me even."

Several participants mentioned how the clothes they wore impacted communication. As this participant described, providers made assumptions about educational and/or financial status based on what they were wearing:

"We have noticed that the folks at [the hospital] treat us differently based upon the clothes that we're wearing. That if I go in my work clothes and my husband goes in his work clothes, we are treated as having a certain level of competency. They didn't go into a great deal to explain things."

To address this, several participants described dressing a particular way so that they would receive the communication and treatment they desired for the circumstances. 
Several participants describe feeling objectified in health care settings. Most often this occurs in comments from providers and staff about appearances. For example, one participant shared a comment a health care provider made about her multiracial daughter:

"'She's just so beautiful. What a unique look. She's going to grow up to look like Beyoncé.' Just weird stuff. Where I'm like, she just looks like any other mixed-race baby. I don't know why she's exotic."

Participants mention such encounters left them feeling uncomfortable or awkward as a result.

\section{Intersectionality}

While the focus of this project was to capture racial microaggressions, participants expressed that they often could not distinguish these from other microaggressions. As one participant described, race alone may not have been the only cause of discrimination but the intersection of multiple marginalized identities:

"I guess it's just one of those things that it's hard for me to necessarily pull out what is race-related and what isn't. I think the thing that kind of has been hitting home most recently has been that I am also a disabled person. ... I get a lot of stigmatizing language around addiction. And I think that that's really interesting because of what I know culturally about the stigma against Native American and Latino people is a lot around this idea that all Natives are drunks, and kind of the same thing is stigmatized around Latinos."

Participants discussed the intersections of gender, age, and race most frequently. However, citizenship, disability, language, and insurance also were mentioned.

\section{DISCUSSION}

Previous work outlined a taxonomy for multiracial microaggressions in clinical behavioral health settings, highlighting how multiracial microaggressions in everyday life parallel those in clinical settings. ${ }^{9}$ While scholars have begun to identify and explore the prevalence and significance of microaggressions in health care settings, ${ }^{2,6,22}$ to our knowledge our work is the first to date to document multiracial patients' experience of these microaggressions and to do so in their own words.
Johnston and Nadal describe a framework of five dominant manifestations of microaggressions for multiracial individuals: exclusion and isolation; exoticization and objectification; mistaken identity; denial of multiracial reality; and pathologizing of identity and experience. ${ }^{9}$ Our work reaffirms that these manifestations exist in health care settings and provides nuance in terms of how they manifest. Regarding exoticization and objectification, this paralleled much of what patients described related to pervasive stereotypes. Patients commonly cited mistaken identity, particularly in electronic health records, and denial of multiracial identity as described in our fixed forms theme.

However, our work also illuminates some key distinctions between the existing taxonomy for multiracial microaggressions and patients' reporting about their experiences in health care settings. First, patients describe exclusion and isolation in more nuanced ways. Arguably similar to people of color generally, some reported feeling different from predominantly white health care providers and staff. A central point that has been unaddressed in previous work is the feeling of being excluded from one's own family by providers. While most participants acknowledged that this is not necessarily different from everyday life, the impact intensified in health care settings. In health care, people often felt a need or desire for family support related to the circumstances of seeking care with a family member - whether in supporting a relative's care or in receiving support during one's own health care. For some, this isolation and exclusion distanced them from decisionmaking and felt disempowering.

Second, pathologizing of identity and experience extended broader than race; this notion manifested as pathologizing health behaviors. Patients expressed provider assumptions about promiscuity, alcohol and drug misuse, diet, and exercise, despite patients reporting otherwise. Furthermore, several expressed provider assumptions about a lack of health literacy.

Third, previous work has not illuminated with the same attention and clarity the impact of the power relationship between patients and providers in dealing with questions that felt intrusive. While patients described instances of feeling uncomfortable or 
awkward, they often responded to providers' questions. A key difference between racial microaggressions experienced outside health care versus inside is power dynamics between patients and providers. Unlike everyday experiences of microaggressions in which one might ignore or disregard a stranger's comment or question, in the context of seeking health care, patients were uncertain as to what questions they felt they needed to supply to guide their health care decision-making versus what they were being asked to satisfy a provider's curiosity. When combined with monoracial assumptions that can exclude family racial dynamics and constrict racial identities, race may have a heighted salience for multiracial patients. Taken together, findings from this study further illustrate and add nuance to the complexity and prevalence of racial microaggressions in health care settings.

This work focuses on the experience of multiracial individuals; however, some of the findings suggest that health care settings may operate under a narrow construct about the meaning of family. This could have broader implications for family-centered care and patient-family-provider communication. Further work should explore this more directly, particularly in pediatrics, obstetrics and gynecology, and geriatrics, areas for which we would expect higher levels of family engagement.

\section{Limitations}

Because of the purposive and snowball sampling strategies employed, we acknowledge some study limitations. First, the majority of our sample identified as college-educated women of reproductive age. This may limit the range and types of racism and microaggressions potentially identified with more diversity in terms of education, gender, and age. Second, as this was a self-selected sample, some of the participants had specialized training or interest in mixed-race studies or public health. In this regard, participants may have been more aware of and able to verbalize their experiences of racism and microaggressions than the general community. Third, we conducted interviews and focus groups with individual patients; while this provided valuable insights into individual experiences, it also highlights an opportunity for future research incorporating multiple family members' perspectives.
Nonetheless, the strength of this study is its internal validity, given the recurring themes identified within and across multiple interviews and focus groups. Furthermore, many of the themes that emerged were consistent with descriptions of racism and racial microaggressions in the health care literature as well as the multiracial literature, contributing to external validity. While this study was exploratory in nature and not meant to be representative of the full diversity of the multiracial patient population, the complexity of race and racism emerging from these findings sheds light on the experience of racial microaggressions for multiracial people and families in health care and may inform efforts to provide culturally responsive patientand family-centered care. Given the dearth of research in this area, this study lays the groundwork for future larger-scale quantitative and mixed-methods studies with a more diverse sample.

\section{Practical Implications}

Several practical implications for improving patient experiences and facilitating systematic improvements in the provision of culturally responsive health care services emerged from this study. First, the findings are particularly relevant to health profession education and training programs. For example, many of the textbooks and materials focused on diversity and cultural competency do not discuss multiraciality, and many educators may not be familiar with teaching such topics. Furthermore, lectures and discussions about the various forms of racism, including microaggressions, are often not approached in detail, if at all, in the medical curricula and need to be more deeply addressed in programs and courses. Studies have found that although social determinants of health and health disparities are increasingly covered in health and medical curricula, students and residents still leave their training underprepared to tackle issues of racism and health upon entering the field; even more problematic, some still hold implicitly or explicitly racist beliefs about people of color. ${ }^{23,24}$

Second, our study findings suggest possible professional development opportunities for currently practicing providers. In terms of efforts to address racial bias and monoracial assumptions, professional development efforts could include partnering with culturally responsive multiracial organizations for training and 
development opportunities. Likewise, antiracism training for health care professionals is necessary, but not sufficient to systematically address various forms of the individual, institutional, and systemic racism that shape health care contexts. Efforts should be made to address the systemic issues that shape patient experiences and health outcomes both within and beyond the health care setting. Furthermore, assessing health care providers' perspectives, knowledge, and experience working with multiracial individuals and families, as well as their knowledge about racial microaggressions, may yield additional insights to help design efforts to facilitate the provision of culturally responsive health care services.

\section{CONCLUSIONS}

In addition to illuminating how racial microaggressions commonly manifest in health care for multiracial people and families, our study findings build on previous work ${ }^{25}$ showing how unexamined provider biases on race, gender and sexuality, weight, and socioeconomic status can impact clinical decisionmaking and perpetuate health disparities. A deeper understanding of patient perspectives about racial microaggressions reveals opportunities to improve patient-provider communication. For multiracial individuals and families, racial microaggressions may have implications for patient engagement in health care and can ultimately impact health and well-being. To help facilitate systematic change, further work is needed to understand the specific mechanisms by which experiences with racial microaggressions in health care settings impact health and well-being among people of color, particularly among multiracial individuals.

\section{Patient-Friendly Recap}

- Multiracial individuals and families provide a unique perspective on patient experiences of racism and racial bias.

- Using a combination of interviews and focus groups, the authors illuminated how racial microaggressions commonly manifest in health care settings.

- Patients' reported experiences may guide improvement in patient-provider communication and redirect the design of culturally responsive care practices.

\section{Acknowledgments}

We acknowledge the Stakeholder and Patient Advisory Group members, who provided feedback in the development of this manuscript. We also would like to acknowledge our participants for their candidness about their health care experiences.

\section{Author Contributions}

Study design: Snyder, Truitt. Data acquisition or analysis: all authors. Manuscript drafting: all authors. Critical revision: all authors.

\section{Conflicts of Interest}

None.

\section{Funding Sources}

This project was supported by a grant (K12HS022982) from the Agency for Healthcare Research and Quality (Rockville, MD). The content is solely the responsibility of the authors and does not necessarily represent the official views of the Agency for Healthcare Research and Quality.

\section{References}

1. Institute of Medicine (US) Committee on Understanding and Eliminating Racial and Ethnic Disparities in Health Care; Smedley BD, Stith AY, Nelson AR (eds). Unequal Treatment: Confronting Racial and Ethnic Disparities in Health Care. Washington, DC: National Academies Press, 2003.

2. Walls ML, Gonzalez J, Gladney T, Onello E. Unconscious biases: racial microaggressions in American Indian health care. J Am Board Fam Med. 2015;28:231-9. CrossRef

3. Pierce C. Offensive mechanisms. In: Barbour FB (ed). The Black Seventies. Boston, MA: Porter Sargent, 1970, pp. 265-82.

4. Pierce CM. Psychiatric problems of the black minority. In: Arieti S (ed). American Handbook of Psychiatry, Volume 2. New York, NY: Basic Books, 1974, pp. 1353-84.

5. PérezHuber L, Solorzano DG. Racial microaggressions as a tool for critical race research. Race Ethn Educ. 2015;18:297-320. CrossRef

6. Almond AL. Measuring racial microaggression in medical practice. Ethn Health. 2017 Aug 1:1-18. CrossRef

7. Pew Research Center. Multiracial in America: proud, diverse and growing in numbers. Published June 11, 2015. http://www. pewsocialtrends.org/2015/06/11/multiracial-in-america/\#. Accessed May 10, 2018.

8. U.S. Census Bureau. U.S. Census Bureau projections show a slower growing, older, more diverse nation a half century from now (news release, December 12, 2012). http://www.census. gov/newsroom/releases/archives/population/cb12-243.html. Accessed May 10, 2018

9. Johnston MP, Nadal KL. Multiracial microaggressions: exposing monoracism in everyday life and clinical practice. In: Sue DW (ed). Microaggressions and Marginality: Manifestations, Dynamics, and Impact. Hoboken, NJ: Wiley, 2010, pp. 123-44.

10. Ahmann E. Tiger Woods is not the only "Cablinasian:" multiethnicity and health care. Pediatr Nurs. 2005;31:125-9.

11. Coyne IT. Sampling in qualitative research. Purposeful and theoretical sampling; merging or clear boundaries? $J A d v$ Nurs. 1997;26:623-30. CrossRef 
12. Harrell SP. A multidimensional conceptualization of racismrelated stress: implications for the well-being of people of color. Am J Orthopsychiatry. 2000;70:42-57. CrossRef

13. Charmaz K. Constructing Grounded Theory: A Practical Guide Through Qualitative Analysis. London, UK: Sage Publications, 2006.

14. Strauss AL, Corbin JM. Basics of Qualitative Research: Grounded Theory Procedures and Techniques. Newbury Park, CA: Sage Publications, 1990.

15. Tolman DL, Brydon-Miller M (eds). From Subjects to Subjectivities: A Handbook of Interpretive and Participatory Methods. New York, NY: New York University Press, 2001.

16. Ayres L, Kavanaugh K, Knafl KA. Within-case and acrosscase approaches to qualitative data analysis. Qual Health Res. 2003;13:871-83. CrossRef

17. Hsieh HF, Shannon SE. Three approaches to qualitative content analysis. Qual Health Res. 2005;15:1277-88. CrossRef

18. Srivastava P, Hopwood N. A practical iterative framework for qualitative data analysis. Int J Qual Methods. 2009;8(1):76-84. CrossRef

19. SocioCultural Research Consultants, LLC. Dedoose Version 7.0.23 (2016), web application for managing, analyzing, and presenting qualitative and mixed method research data. www. dedoose.com. Accessed May 10, 2018.
20. Tran AG, Miyake ER, Martinez-Morales V, Csizmadia A. "What are you?" Multiracial individuals' responses to racial identification inquiries. Cultur Divers Ethnic Minor Psychol. 2016;22:26-37. CrossRef

21. Tashiro CJ. Standing on Both Feet: Voices of Older Mixed Race Americans. Boulder, CO: Paradigm Publishers, 2012.

22. Bleich MR. Microaggression and its relevance in health care. J Contin Educ Nurs. 2015;46:487-8. CrossRef

23. Hoffman KM, Trawalter S, Axt JR, Oliver MN. Racial bias in pain assessment and treatment recommendations, and false beliefs about biological differences between blacks and whites. Proc Natl Acad Sci U S A. 2016;113:4296-301. CrossRef

24. Okwerekwu JA. The patient called me 'colored girl.' The senior doctor training me said nothing. STAT. Published April 11, 2016. https://www.statnews.com/2016/04/11/racismmedical-education/. Accessed May 10, 2018.

25. Dovidio JF, Penner LA, Albrecht TL, Norton WE, Gaertner SL, Shelton JN. Disparities and distrust: the implications of psychological processes for understanding racial disparities in health and health care. Soc Sci Med. 2008;67:478-86. CrossRef

(C) 2018 Aurora Health Care, Inc. 